\title{
Stage IVA Nasopharyngeal Keratinizing Squamous Cell Carcinoma AJCC v7
}

National Cancer Institute

\section{Source}

National Cancer Institute. Stage IVA Nasopharyngeal Keratinizing Squamous Cell

Carcinoma AJCC V7. NCI Thesaurus. Code C6137.

Stage IVA includes: (T4, N0, M0); (T4, N1, M0); (T4, N2, M0). T4: Nasopharyngeal cancer with intracranial extension and/or involvement of cranial nerves, hypopharynx, orbit, or with extension to the infratemporal fossa/masticator space. N0: No regional lymph node metastasis. N1: Nasopharyngeal cancer with unilateral metastasis in cervical lymph node(s), $6 \mathrm{~cm}$ or less in greatest dimension, above the supraclavicular fossa, and/or unilateral or bilateral, retropharyngeal lymph nodes, $6 \mathrm{~cm}$ or less in greatest dimension. Midline nodes are considered ipsilateral nodes. N2: Nasopharyngeal cancer with bilateral metastasis in cervical lymph node(s), $6 \mathrm{~cm}$ or less in greatest dimension, above the supraclavicular fossa. Midline nodes are considered ipsilateral nodes. M0: No distant metastasis. (AJCC 7th ed.) 